J. J. Meier • B. Gallwitz • M. Askenas $\cdot$ K. Vollmer •

C. F. Deacon · J. J. Holst • W. E. Schmidt • M. A. Nauck

\title{
Secretion of incretin hormones and the insulinotropic effect of gastric inhibitory polypeptide in women with a history of gestational diabetes
}

Received: 25 February 2005 / Accepted: 5 April 2005 / Published online: 12 July 2005

(C) Springer-Verlag 2005

\begin{abstract}
Aims/hypothesis: The insulinotropic effect of gastric inhibitory polypeptide (GIP) is reduced in patients with type 2 diabetes and around $50 \%$ of their first-degree relatives under hyperglycaemic conditions. It is unknown whether this is a result of a specific defect in GIP action or of a general reduction in beta cell function. Moreover, impaired secretion of glucagon-like peptide 1 (GLP-1) has been described in patients with type 2 diabetes. Therefore, we studied the insulinotropic effect of GIP in women with previous gestational diabetes (pGDM) under euglycaemic fasting conditions and during a hyperglycaemic clamp experiment. The secretion of GIP and GLP-1 was assessed following oral glucose ingestion. Materials and methods:
\end{abstract}

J. J. Meier · B. Gallwitz · M. Askenas · K. Vollmer

W. E. Schmidt

Department of Medicine I,

St Josef Hospital,

Ruhr-University of Bochum,

Bochum, Germany

J. J. Meier ( $\square)$

Larry Hillblom Islet Research Center,

UCLA David Geffen School of Medicine,

900A Weyburn Place N.,

Los Angeles, CA 90095, USA

e-mail: jmeier@mednet.ucla.edu

Tel.: +1-310-2067236

Fax: $+1-310-2065368$

B. Gallwitz

Department of Medicine IV,

University of Tübingen,

Tübingen, Germany

C. F. Deacon · J. J. Holst

Department of Medical Physiology,

The Panum Institute,

University of Copenhagen,

Copenhagen, Denmark

M. A. Nauck

The Diabetes Centre,

Bad Lauterberg, Germany
On separate occasions we performed an OGTT and administered an i.v. bolus of $20 \mathrm{pmol} \mathrm{GIP} / \mathrm{kg}$ body weight in 20 women with pGDM and 20 control women. An additional hyperglycaemic clamp experiment $(140 \mathrm{mg} / \mathrm{dl}$ [7.8 mmol/l] over $120 \mathrm{~min})$ with i.v. infusion of GIP (2 pmol kg-1 $\min ^{-1} ; 30-90 \mathrm{~min}$ ) was performed in 14 women in each group. Capillary and venous blood samples were drawn for the measurement of glucose (glucose oxidase), insulin, C-peptide, GIP and GLP-1 (specific immunoassays). Indices of insulin sensitivity and beta cell function were calculated. Statistical analyses were carried out using repeated measures ANOVA. Results: Following oral glucose ingestion, plasma glucose, insulin and C-peptide concentrations increased to higher levels in the women with pGDM than in the control women $(p<0.05)$. The women with pGDM were characterised by a higher degree of insulin resistance than the control women $(p=0.007$ for the Matsuda index), but showed no overt defects in glucosestimulated insulin secretion ( $p=0.40$ for the insulinogenic index following i.v. glucose). The secretion of GLP-1 and GIP was not different between the groups $(p=0.87$ and $p=0.57$, respectively). The insulin secretory response to GIP administration was similar in the two groups both after GIP bolus administration and during the hyperglycaemic clamp experiment ( $p=0.99$ and $p=0.88$, respectively). A hyperbola-like relationship was found between the degree of insulin sensitivity (Matsuda index) and the insulin secretory response to GIP and i.v. glucose administration. Conclusions/interpretation: These results do not support the hypothesis of an early defect in GIP action as a risk factor for subsequent development of diabetes in women with previous gestational diabetes. The inverse relationship between insulin resistance and the insulin secretory response to glucose or GIP suggests that beta cell secretory function in response to different stimuli increases adaptively when insulin sensitivity is diminished.

Keywords Gastric inhibitory polypeptide - Gestational diabetes - Glucose-dependent insulinotropic polypeptide . Incretin hormones - Insulin secretion - Type 2 diabetes 
Abbreviations GIP: gastric inhibitory polypeptide · GLP-1: glucagon-like peptide 1 - HOMA: homeostasis model assessment $\cdot$ pGDM: previous gestational diabetes

\section{Introduction}

The incretin effect describes the phenomenon that oral ingestion of glucose elicits greater increases in insulin secretion than comparable amounts of glucose administered via the intravenous route $[1,2]$. This effect is mediated by the secretion of peptide hormones from the gut, which stimulate insulin secretion $[2,3]$. The two known incretin hormones are gastric inhibitory polypeptide (GIP; also referred to as glucose-dependent insulinotropic polypeptide) and glucagon-like peptide 1 (GLP-1) $[4,5]$.

A reduced incretin effect is characteristic of patients with type 2 diabetes [6], and two different defects in the entero-insular axis have been described in these patients. Firstly, the insulinotropic effect of GIP is markedly reduced in patients with type 2 diabetes, while the effectiveness of GLP-1, although decreased, is preserved to a much higher degree $[7,8]$. Secondly, the secretion of GLP-1 is impaired in patients with type 2 diabetes [9, 10], while GIP secretion appears to be more or less normal [10]. To clarify whether the loss of GIP effect in type 2 diabetes is a result of a primary, possibly genetically determined, defect or whether it develops later in the pathogenesis of type 2 diabetes, we previously studied the insulinotropic effects of GIP in a cohort of first-degree relatives of type 2 diabetes patients during a hyperglycaemic clamp [11]. Under these conditions, around $50 \%$ of the relatives showed defects in GIP-induced insulin secretion, reminiscent of the secretion pattern observed in patients with type 2 diabetes. These results led to the assumption of a primary, possibly genetically determined, defect in GIP action that confers the risk of developing type 2 diabetes $[11,12]$. However, the first-degree relatives included in the study were also characterised by an impairment in glucose-induced insulin secretion [11], meaning that it was difficult to distinguish between an impairment in glucose-stimulated insulin secretion and a specific defect in GIP action.

Women with previous gestational diabetes (pGDM) represent another group at high risk of developing type 2 diabetes [13-15], and defects in insulin secretion and insulin action have been described in pGDM women from different geographic and ethnic backgrounds [15]. In addition, it has been suggested that type 2 diabetes in these women develops with a different genetic basis than in other high-risk cohorts, such as in first-degree relatives [15]. We saw this as an opportunity to investigate the insulinotropic effect of GIP as well as the secretion of incretin hormones in this pre-diabetic cohort. To distinguish between defects in beta cell function in response to intravenous glucose and those in response to intravenous GIP, the insulinotropic effect of GIP was examined both under euglycaemic fasting conditions and during a hyperglycaemic clamp.

\section{Subjects and methods}

\section{Study protocol}

Prior to the study, the study protocol was approved by the ethics committee of the medical faculty of the Ruhr-University of Bochum on 1 January 2001 (registration number 1615). Written informed consent was obtained from all participants.

\section{Subjects}

Twenty women with pGDM and 20 control women with no history of diabetes in themselves or their first-degree relatives were studied. Participants were randomly recruited by local advertisements as well as by contacting local obstetrics departments. In the women with pGDM, the disease had been diagnosed by their respective gynaecologists. Since uniform guidelines for the diagnosis of gestational diabetes had not been established in all centres at that time, the diagnosis of gestational diabetes was based on an OGTT (15 cases) or on elevated fasting glucose levels (five cases). The diagnosis was made during gestational week $26 \pm 6($ mean \pm SD). The average time interval between pregnancy and the experiments was $4.1 \pm 6.5$ years. Four women required insulin treatment during pregnancy, whereas hyperglycaemia was controlled by a dietary regimen in the other cases. None of the women required hypoglycaemic treatment between delivery and the time of study commencement. The number of previous gestations (prior to the occurrence of gestational diabetes) was $1.8 \pm$ 1.1. Eight women with pGDM had a first-degree relative with type 2 diabetes, and an additional three women in this group had a second-degree relative with type 2 diabetes. Two women with pGDM, but no control women, had had spontaneous abortions during previous pregnancies. Hypertension was present in one woman with pGDM, but was absent in all control subjects. No subject had overt hyperlipidaemia. None of the women received any medication with a known effect on glucose homeostasis.

Blood was drawn in the fasting state from all women for measurement of standard haematological and clinical chemistry parameters. None of the women had anaemia (haemoglobin $<11 \mathrm{~g} / \mathrm{dl}[110 \mathrm{~g} / 1]$ ), an elevation in liver enzymes (alanine aminotransferase, aspartate aminotransferase, alkaline phosphatase or $\gamma$-glutaryltransferase) to higher activities than double the respective normal value, or elevated creatinine concentrations $(>1.5 \mathrm{mg} / \mathrm{dl}[132.6 \mu \mathrm{mol} / 1])$. Body height, weight and waist and hip circumference were measured to calculate body mass index and waist-to-hip 
Table 1 Characteristics of the women with previous gestational diabetes (pGDM) and of control subjects

Data are means $\pm \mathrm{SD}$

\begin{tabular}{lccc}
\hline Parameter & Women with pGDM & Control women & $p$ value (ANOVA) \\
\hline Age (years) & $36.2 \pm 5.1$ & $37.5 \pm 7.9$ & 0.57 \\
Weight $(\mathrm{kg})$ & $71.8 \pm 13.7$ & $63.1 \pm 9.7$ & 0.03 \\
Height $(\mathrm{cm})$ & $167 \pm 6$ & $169 \pm 4$ & 0.25 \\
BMI $\left(\mathrm{kg} / \mathrm{m}^{2}\right)$ & $25.9 \pm 5.1$ & $22.2 \pm 3.2$ & 0.01 \\
WHR & $0.82 \pm 0.08$ & $0.78 \pm 0.07$ & 0.081 \\
Birthweight of infants $(\mathrm{g})$ & $3,615 \pm 661$ & $3,165 \pm 289$ & 0.046 \\
Systolic BP $(\mathrm{mmHg})$ & $114 \pm 15$ & $110 \pm 10$ & 0.40 \\
Diastolic BP $(\mathrm{mmHg})$ & $72 \pm 12$ & $71 \pm 11$ & 0.68 \\
Total cholesterol $(\mathrm{mmol} / \mathrm{l})$ & $5.02 \pm 0.68$ & $5.05 \pm 0.80$ & 0.92 \\
HDL-cholesterol $(\mathrm{mmol} / \mathrm{l})$ & $1.21 \pm 0.49$ & $1.53 \pm 0.43$ & 0.041 \\
LDL-cholesterol $(\mathrm{mmol} / \mathrm{l})$ & $3.28 \pm 0.97$ & $3.16 \pm 0.82$ & 0.69 \\
Triglycerides $(\mathrm{mmol} / \mathrm{l})$ & $1.29 \pm 0.56$ & $0.95 \pm 0.57$ & 0.07 \\
HbA $\mathrm{A}_{1}$ c $\left.\%\right)$ & $5.5 \pm 0.4$ & $5.6 \pm 0.6$ & 0.71 \\
\hline
\end{tabular}

ratio (Table 1). Blood pressure was taken according to the Riva-Rocci method.

\section{Study design}

All women first participated in a screening visit. Patient history was recorded, a clinical examination was performed, and haematological and clinical chemistry parameters were screened in the fasting state. Urine was tested for $\beta$-human chorionic gonadotrophin to exclude the possibility of pregnancy. If subjects met the inclusion criteria, they were recruited for the following tests:

(1) an oral glucose tolerance test. After drawing two basal ( -15 and $0 \mathrm{~min}$ ) capillary and venous blood samples, a glucose drink (75 g; Boehringer OGT, Mannheim, Germany) was ingested within 5 min. Capillary and venous blood samples were obtained after 30, 60, 90 and $120 \mathrm{~min}$;

(2) intravenous bolus administration of GIP. After drawing basal venous and capillary blood samples ( -5 and 0 $\mathrm{min})$, synthetic human GIP (20 pmol/kg body weight) was injected intravenously into a large forearm vein, as described previously [16]. Venous blood samples from a cannula placed into a contralateral forearm vein, and capillary samples, were obtained at 1, 3, 5, 10, 15, 20 and $30 \mathrm{~min}$. Both experiments were carried out in random order with an average interval of $16 \pm 23$ days (mean $\pm \mathrm{SD}$ ) separating the tests. All women were contacted again $12 \pm 3$ months after these initial experiments and asked to participate in an additional test to study the insulinotropic effect of GIP under hyperglycaemic conditions. Fourteen of the originally studied 20 women in each group participated in this additional experiment. A hyperglycaemic clamp test designed to achieve a steady capillary plasma glucose concentration of $7.8 \mathrm{mmol} / \mathrm{l}(140 \mathrm{mg} / \mathrm{dl})$ was performed in 28 women (14 in each group). The clamp procedure was started by injecting $40 \%$ glucose as a bolus and maintained for $120 \mathrm{~min}$ by infusing glucose $(20 \%$ in water, $\mathrm{w} / \mathrm{v}$ ) as appropriate, based on glucose measurements performed every $5 \mathrm{~min}$. Glucose was infused alone for the first $30 \mathrm{~min}$ to estimate glucose-stimulated insulin secretion. From 30 to 90 min, GIP was administered intravenously at an infusion rate of $2.0 \mathrm{pmol} \mathrm{kg}^{-1} \mathrm{~min}^{-1}$, as previously described [11].

Peptides

Synthetic GIP was purchased from PolyPeptide Laboratories (Wolfenbüttel, Germany) and processed for infusion as described previously [11].

\section{Experimental procedures}

The tests were performed in the morning after an overnight fast. The subjects were in a supine position with the upper body lifted approximately $30^{\circ}$ throughout the experiments. Two forearm veins were punctured using a Teflon cannula (Moskito 123, 18 gauge; Vygon, Aachen, Germany) and kept patent using $0.9 \% \mathrm{NaCl}$ (for blood sampling and for glucose and GIP administration, respectively). Both ear lobes were made hyperaemic using Finalgon (Nonivamid 4 $\mathrm{mg} / \mathrm{g}$, Nicoboxil $25 \mathrm{mg} / \mathrm{g}$ ).

\section{Blood specimens}

Venous blood was drawn into chilled tubes containing EDTA and aprotinin (Trasylol; 20,000 Kallikrein Inhibitor Units $/ \mathrm{ml}, 200 \mu \mathrm{l}$ per $10 \mathrm{ml}$ blood; Bayer, Leverkusen, Germany) and kept on ice. After centrifugation at $4^{\circ} \mathrm{C}$, plasma for hormone analyses was kept frozen at $-28^{\circ} \mathrm{C}$. This procedure has previously been shown to prevent in vitro degradation of incretin hormones in human plasma samples [17]. Capillary blood samples (approximately 100 4 ) were added to NaF (Microvette CB 300; Sarstedt, Nümbrecht, Germany) for the immediate measurement of glucose. 


\section{Laboratory measurements}

Glucose was measured by the glucose oxidase method using a Glucose Analyser 2 (Beckman Instruments, $\mathrm{Mu}$ nich, Germany).

Insulin was measured as described previously [11] using an insulin microparticle enzyme immunoassay (IMx Insulin; Abbott Laboratories, Wiesbaden, Germany). Intraassay coefficients of variation were approximately $4 \%$.

C-peptide was measured as described previously [11] using an ELISA from Dako (Cambridge, UK). Intra-assay coefficients of variation were 3.3 to $5.7 \%$; inter-assay variation was 4.6 to $5.7 \%$. Human insulin and C-peptide were used as standards.

GIP immunoreactivity was measured using two different assays specific for the $\mathrm{C}$ terminus or the $\mathrm{N}$ terminus of the peptide, as previously described [18]. The C-terminal assay involving antiserum R65 reacted fully with intact GIP (1-42) and the truncated metabolite (3-42) but not with the so-called 8-kDa GIP, of which the chemical nature and molecular relation to GIP is uncertain. The assay had a detection limit of less than $2 \mathrm{pmol} / \mathrm{l}$ and an intraassay variation of approximately $6 \%$. The $\mathrm{N}$-terminal assay measures the concentration of intact GIP (1-42) using antiserum 98171. The cross-reactivity with GIP (3-42) was less than $0.1 \%$. The lower detection limit of the assay was approximately $5 \mathrm{pmol} / \mathrm{l}$. Intra-assay variation was less than $6 \%$, and inter-assay variation was approximately 8 and $12 \%$ for 20 and $80 \mathrm{pmol} / 1$ standards, respectively. For both assays, human GIP (Peninsula Laboratories Europe, St Helens, UK) was used as standard, and radiolabelled GIP was obtained from Amersham Pharmacia Biotech (Aylesbury, UK).

GLP-1 immunoreactivity was determined using a Cterminal radioimmunoassay that measures the sum of the intact peptide and the primary metabolite GLP-1 (9-36 amide), using the antiserum 89390 and synthetic GLP-1 (7-36 amide) as standard. This assay is particularly suitable for estimating the rate of GLP-1 secretion and cross-reacts less than $0.01 \%$ with C-terminally truncated fragments and $83 \%$ with GLP-1 (9-36 amide). The detection limit was 3 $\mathrm{pmol} / \mathrm{l}$. Intra-assay and inter-assay coefficients of variation were less than 6 and $15 \%$, respectively, at $40 \mathrm{pmol} / 1$ [17].

Valine pyrrolidide $(0.01 \mathrm{mmol} / \mathrm{l}$, final concentration $)$ was added to the assay buffers to prevent $\mathrm{N}$-terminal degradation of GIP and GLP-1 during the assay incubation periods.

\section{Calculations}

Insulin resistance/sensitivity was calculated using homeostasis model assessment (HOMA), which is based on fasting insulin and glucose concentrations [19], and the Matsuda insulin sensitivity index, which takes into account mean insulin and glucose levels following oral glucose stimulation [20]. The latter index has recently been specifically validated in women with gestational diabetes [21] and was therefore used for subsequent analyses.
Beta cell function was estimated using three different approaches. The HOMA beta cell function index estimates beta cell function using fasting insulin and glucose levels [19]. The insulinogenic index used $30 \mathrm{~min}$ after oral glucose ingestion provides a measure of early insulin secretion in response to oral glucose stimulation [22]. To assess the insulin secretory response to intravenous glucose, a similar index was calculated from the glucose and insulin plasma concentrations measured after the administration of intravenous glucose during the clamp experiment. The following equation was used: insulinogenic index clamp $15^{\prime}=\left(\right.$ insulin $_{\text {clamp }} \quad 15^{\prime}-$-insulin $\left._{\text {clamp }} \quad 0^{\prime}\right) /\left(\right.$ glucose $_{\text {clamp }} \quad 15^{\prime}$ - glucose $_{\text {clamp } 0^{\prime}}$ ).
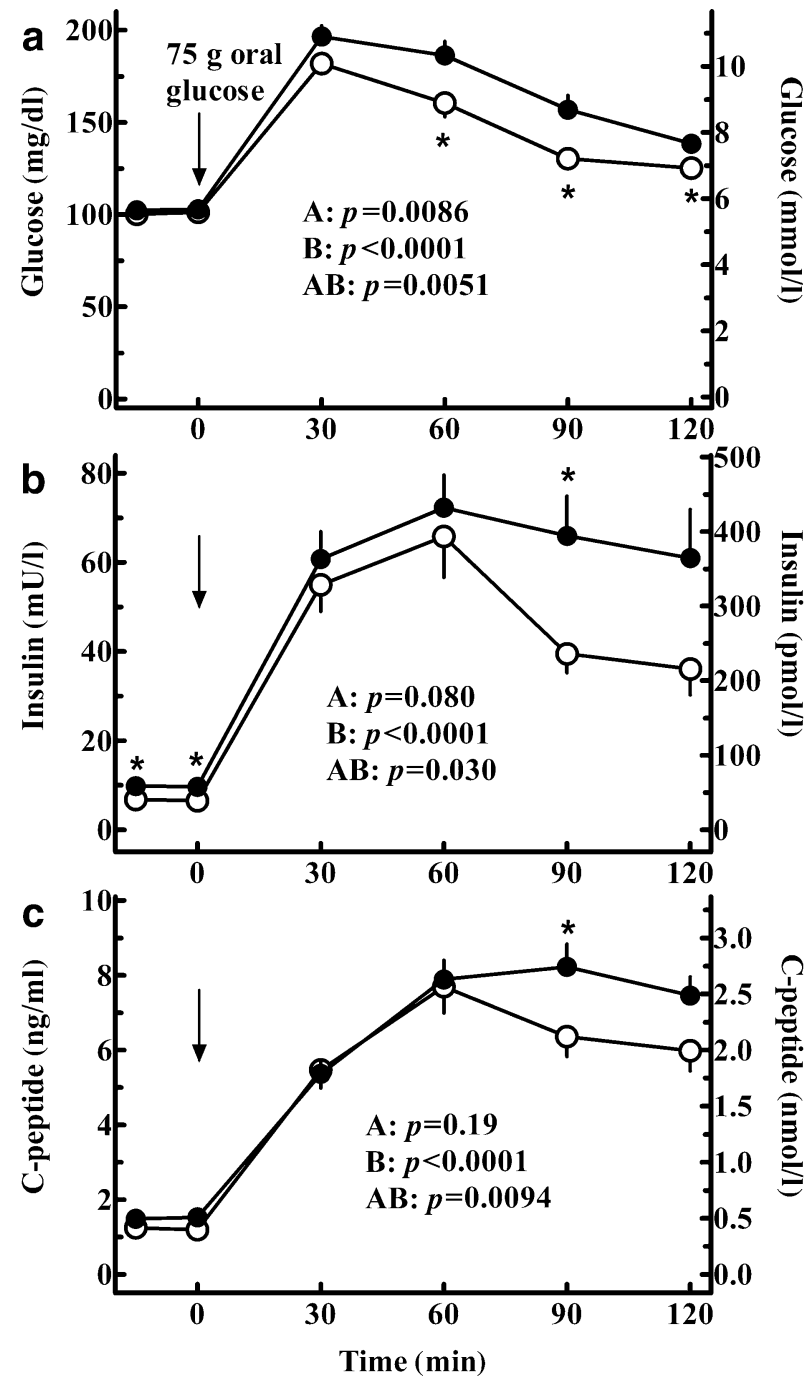

Fig. 1 Plasma concentrations of glucose (a), insulin (b) and Cpeptide (c) after the ingestion of $75 \mathrm{~g}$ oral glucose in 20 women with a history of gestational diabetes (filled symbols) and 20 control women (open symbols). Data are presented as means \pm SEM; $p$ values were calculated using repeated measures ANOVA and denote $A$ differences between the groups, $B$ differences over time, and $A B$ differences owing to the interaction of group and time. Asterisks indicate significant differences at individual time points $(p<0.05$ by one-way ANOVA) 

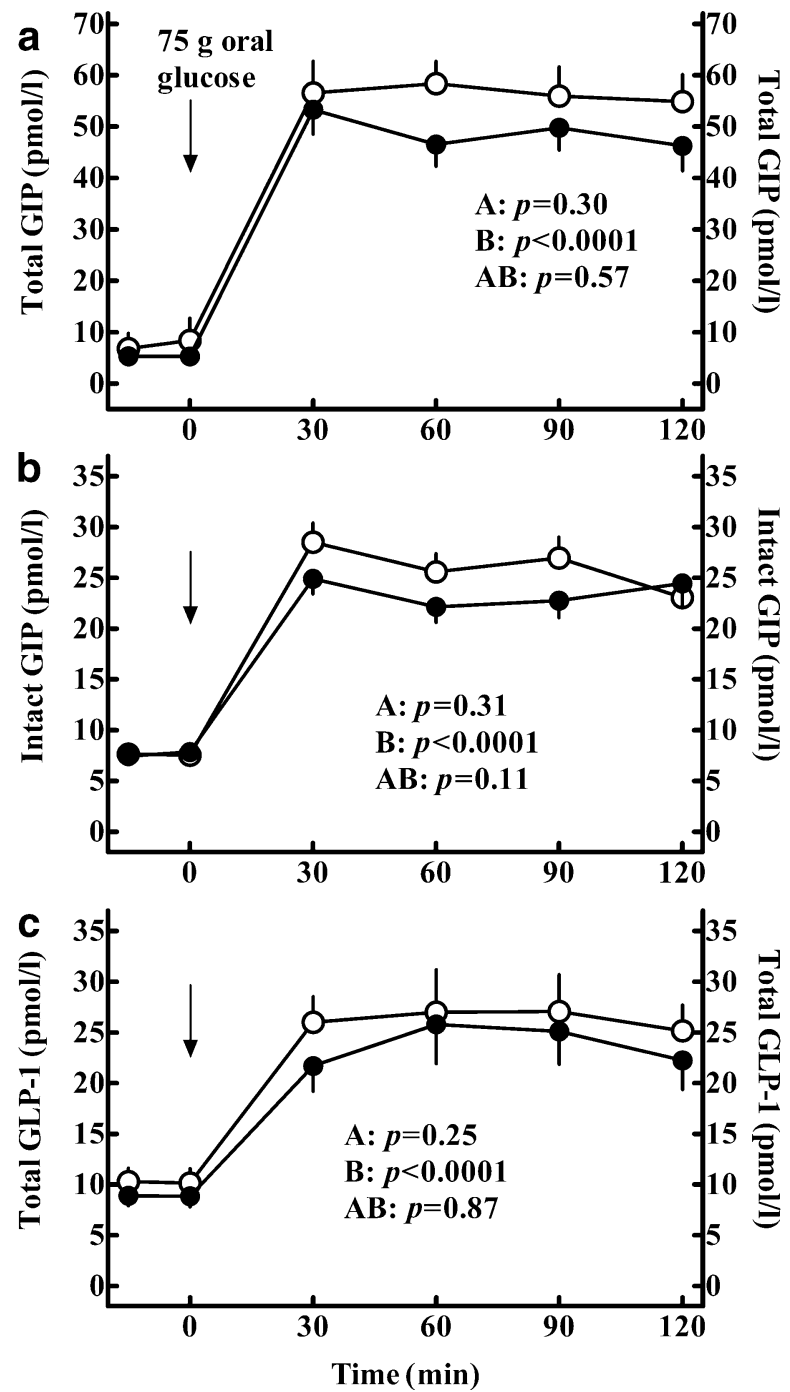

Fig. 2 Plasma concentrations of total GIP (1-42 plus split products; a), intact GIP (1-42; b) and total GLP-1 (7-36 amide plus split products; c) after the ingestion of $75 \mathrm{~g}$ oral glucose in 20 women with a history of gestational diabetes (filled symbols) and 20 control women (open symbols). Data are presented as means \pm SEM; $p$ values were calculated using repeated measures ANOVA and denote $A$ differences between the groups, $B$ differences over time, and $A B$ differences owing to the interaction of group and time

Plasma insulin levels at 5 min were chosen to assess the insulin secretory response to the GIP bolus administration, because at this time point the stimulation of insu- lin secretion was maximal. Plasma insulin concentrations measured after 15 min of the hyperglycaemic clamp experiment were chosen as a marker of the acute insulin response to intravenous glucose administration. Since GIP plasma concentrations reached peak levels after $90 \mathrm{~min}$, plasma insulin concentrations at this time point were used to assess the beta cell response to the combined stimulation with GIP and glucose. For integrated incremental responses of glucose and insulin, the AUC was calculated using the trapezoidal method (baseline subtracted).

\section{Statistical analysis}

Results are reported as means \pm SEM. The following parameters were defined as main outcome variables: (1) total GLP-1 plasma concentrations after $120 \mathrm{~min}$ of the OGTT; (2) total GIP plasma concentrations after $120 \mathrm{~min}$ of the OGTT; (3) plasma insulin concentrations 5 min after GIP bolus administration; and (4) plasma insulin concentrations after $90 \mathrm{~min}$ of the hyperglycaemic clamp experiment. All variables were normally distributed according to the nonparametric Kolmogorov-Smirnov test. Continuous variables were compared using repeated measures ANOVA and Statistica version 5.0 software (Statsoft Europe, Hamburg, Germany). This analysis provides $p$ values for differences between groups/experiments (A), differences over time (B) and the interaction of group/experiment with time (AB). If a significant interaction of treatment and time was documented $(p<0.05)$, values at single time points were compared by one-way ANOVA. A $p$ value of less than 0.05 was taken to indicate a significant difference. Regression analyses were performed using GraphPad Prism, version 3.0 (San Diego, CA, USA).

\section{Results}

Subject characteristics

Both groups were matched for age and $\mathrm{HbA}_{1} \mathrm{c}$ levels (Table 1). The women with pGDM had a higher body mass index than the control women $(p=0.01)$. The birthweight of the infants was higher in the women with pGDM $(p=0.046)$. No differences in the prevalence of hypertension or hyperlipidaemia were found between the groups, but the women with pGDM were characterised by some-
Table 2 Insulin sensitivity/ resistance and beta cell function calculated according to different indices based on fasting measurements or an oral glucose tolerance test

Data are means \pm SEM

\begin{tabular}{lllll}
\hline Index & Women with pGDM & Control women & $p$ value (ANOVA) & Reference \\
\hline Insulin sensitivity/resistance & & & & \\
HOMA & $2.47 \pm 0.29$ & $1.67 \pm 0.18$ & 0.029 & {$[19]$} \\
Matsuda index & $3.64 \pm 0.29$ & $5.62 \pm 0.61$ & 0.0070 & {$[20]$} \\
Insulin secretion & & & & \\
HOMA beta cell function & $92.1 \pm 12.6$ & $66.1 \pm 7.3$ & 0.09 & {$[19]$} \\
Insulinogenic index & $60.1 \pm 6.7$ & $65.6 \pm 7.5$ & 0.64 & {$[22]$} \\
Insulinogenic index (30') & $23.3 \pm 4.5$ & $17.6 \pm 4.5$ & 0.40 & - \\
\hline
\end{tabular}


what lower HDL cholesterol concentrations $(p=0.041)$ and tended to have higher triglyceride levels $(p=0.07$; Table 1$)$.

\section{Oral glucose tolerance test}

Fasting glucose concentrations were similar in the two groups (Fig. 1a). After the ingestion of $75 \mathrm{~g}$ glucose, plasma glucose concentrations reached significantly higher levels in the women with pGDM from 60 to $120 \mathrm{~min}$ ( $p=0.0051$, Fig. 1a). Among the women with pGDM, 13 had normal oral glucose tolerance, four had impaired glucose tolerance and three had impaired fasting glucose. In contrast, oral glucose tolerance was normal in all control women according to American Diabetes Association guidelines [23].
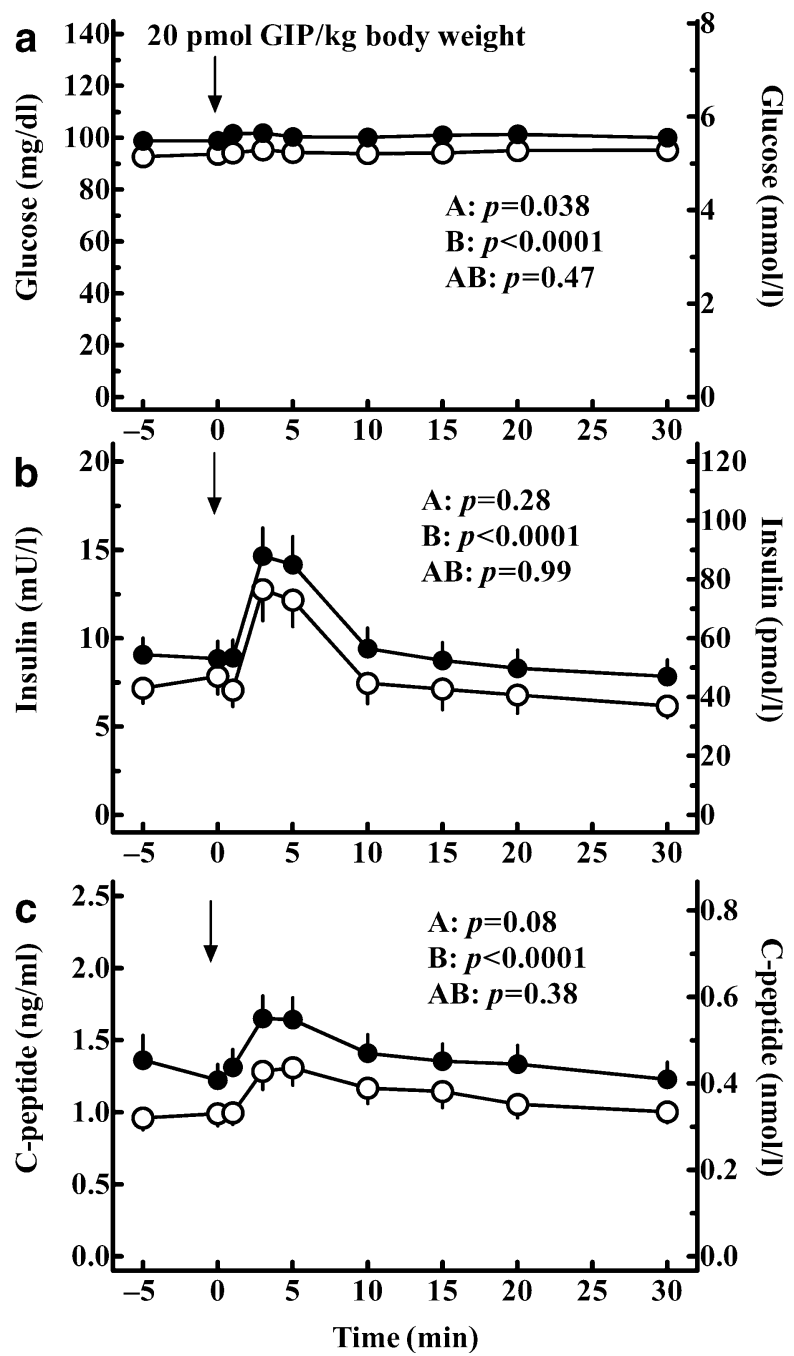

Fig. 3 Plasma concentrations of glucose (a), insulin (b) and Cpeptide (c) after the intravenous bolus administration of $20 \mathrm{pmol}$ $\mathrm{GIP} / \mathrm{kg}$ body weight in 20 women with a history of gestational diabetes (filled symbols) and 20 control women (open symbols). Data are presented as means \pm SEM; $p$ values were calculated using repeated measures ANOVA and denote $A$ differences between the groups; $B$ differences over time, and $A B$ differences owing to the interaction of group and time
Fasting insulin concentrations were already somewhat elevated in the women with pGDM compared with the control women $(p<0.05)$, and these differences became more apparent between 90 and 120 min after glucose ingestion ( $p=0.03$; Fig. 1b). Likewise, plasma C-peptide levels were significantly higher at these time points (but not in the fasting state) in the women with pGDM ( $p=$ 0.0094; Fig. 1c).

Fasting concentrations of both incretin hormones were not different between the groups (Fig. 2). The glucose load led to a significant increase in the secretion of GIP and GLP-1 ( $p<0.001$; Fig. 2). Peak concentrations were $60.4 \pm$ 4.7 and $65.5 \pm 5.7 \mathrm{pmol} / \mathrm{l}$ for total GIP $(p=0.51), 29.8 \pm 1.7$ and $31.2 \pm 1.8 \mathrm{pmol} / 1$ for intact GIP $(p=0.57)$ and $30.7 \pm 4.0$ and $33.0 \pm 4.1 \mathrm{pmol} / 1$ for total GLP-1 $(p=0.70)$, for women with pGDM and control women, respectively. There was no difference in the time course of incretin secretion between the groups (Fig. 2).

\section{Indices of insulin resistance and beta cell function}

The degree of insulin resistance was significantly higher in the women with pGDM than in the control women, according to HOMA and the Matsuda index $(p<0.05$;
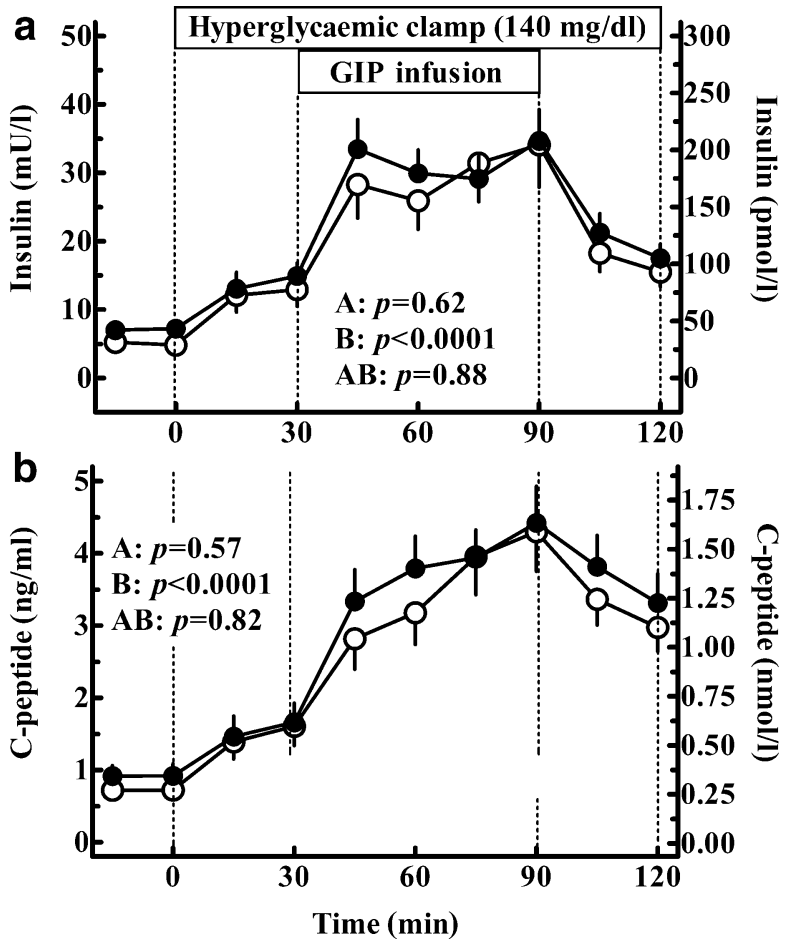

Fig. 4 Plasma concentrations of insulin (a) and C-peptide (b) during a hyperglycaemic clamp experiment with the administration of GIP in 14 women with a history of gestational diabetes (filled symbols) and 14 control women (open symbols). The hyperglycaemic clamp experiment was maintained from 0 to $120 \mathrm{~min}$. From 30 to $90 \mathrm{~min}$, synthetic human GIP was infused intravenously at an infusion rate of $2 \mathrm{pmol} \mathrm{kg}^{-1} \mathrm{~min}^{-1}$. Data are presented as means \pm $\mathrm{SEM} ; p$ values were calculated using repeated measures ANOVA and denote $A$ differences between the groups, $B$ differences over time; and $A B$ differences owing to the interaction of group and time 
Table 2). These differences remained valid when the women who had not participated in the hyperglycaemic clamp experiment were excluded from the analyses (details not shown). In contrast, beta cell function was comparable in the two groups by all measures applied (Table 2).

\section{GIP bolus administration}

The intravenous bolus administration of GIP in the fasting state led to a similar rise in GIP plasma concentrations in the two groups ( $p=0.97$ for total GIP and $p=0.20$ for intact GIP; details not shown). Plasma glucose levels slightly increased after GIP bolus administration ( $p<$ 0.0001; Fig. 3a), while insulin and C-peptide levels increased significantly $(p<0.0001)$. There were no differences between the groups in the plasma concentrations of glucose, insulin or C-peptide following GIP administration ( $p=0.47, p=0.99$ and $p=0.38$, respectively; Fig. 3 ). However, plasma glucose concentrations as well as insulin and C-peptide levels were higher by trend in the women with pGDM during the entire experimental period, compatible with a higher degree of insulin resistance.

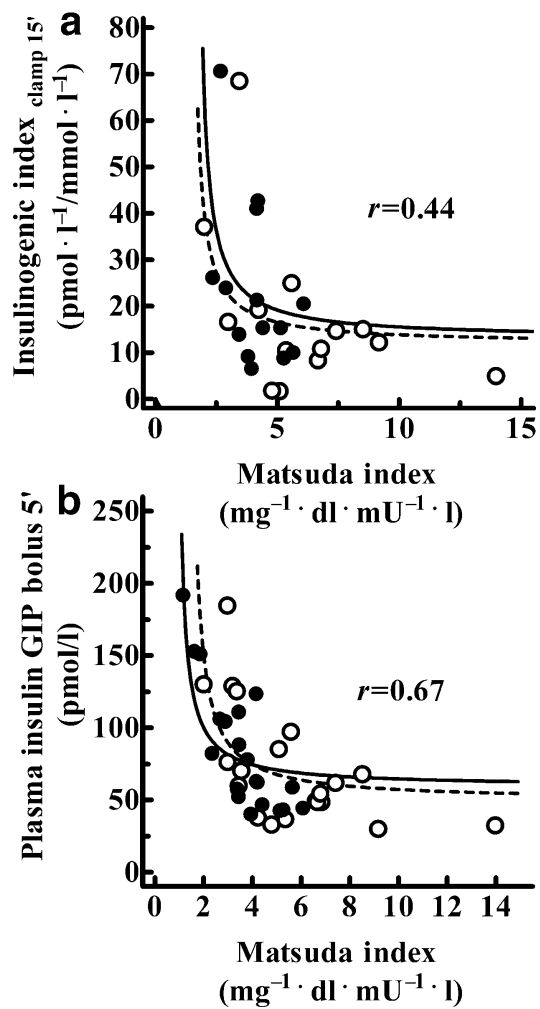

Fig. 5 The relationship between the individual degree of insulin sensitivity, as derived from the Matsuda index, and the insulinogenic index calculated during the intravenous administration of glucose alone (after 15 min of the clamp experiment; (a) and during the combined stimulation with intravenous glucose and GIP (after 90 min of the clamp experiment; (b) in 14 women with a history of gestational diabetes (filled symbols) and 14 control women (open symbols). $r$, correlation coefficient, calculated by non-linear regression analysis. Straight lines, women with pGDM; dashed lines, control women
Hyperglycaemic clamp experiment with GIP infusion

During the hyperglycaemic clamp experiment, similar plasma glucose levels were reached in the two groups. Mean plasma glucose concentrations were $140.1 \pm 0.7$ and $139.0 \pm$ $0.8 \mathrm{mg} / \mathrm{dl}[7.8 \pm 0.04$ and $7.7 \pm 0.04 \mathrm{mmol} / \mathrm{l}]$ in women with pGDM and control women, respectively $(p=0.12)$. The mean glucose infusion rates required to maintain hyperglycaemia were $4.16 \pm 0.38$ and $4.76 \pm 0.45 \mathrm{mg} \mathrm{kg}^{-1} \mathrm{~min}^{-1}$ in women with pGDM and control women, respectively $(p=0.32)$. The intravenous infusion of GIP led to similar plasma levels for total and intact GIP in the two groups ( $p=0.13$ and $p=0.40$, respectively; details not shown).

Plasma concentrations of insulin and C-peptide increased to a similar extent in the two groups during the hyperglycaemic clamp period without GIP infusion (1530 min; Fig. 4). Exogenous GIP administration further augmented insulin secretion $(p<0.0001)$. However, insulin secretory responses to GIP administration during the hyperglycaemic clamp were not different between the groups ( $p=0.88$ and $p=0.82$ for insulin and C-peptide, respectively; Fig. 4). Integrated AUCs of insulin concentra-
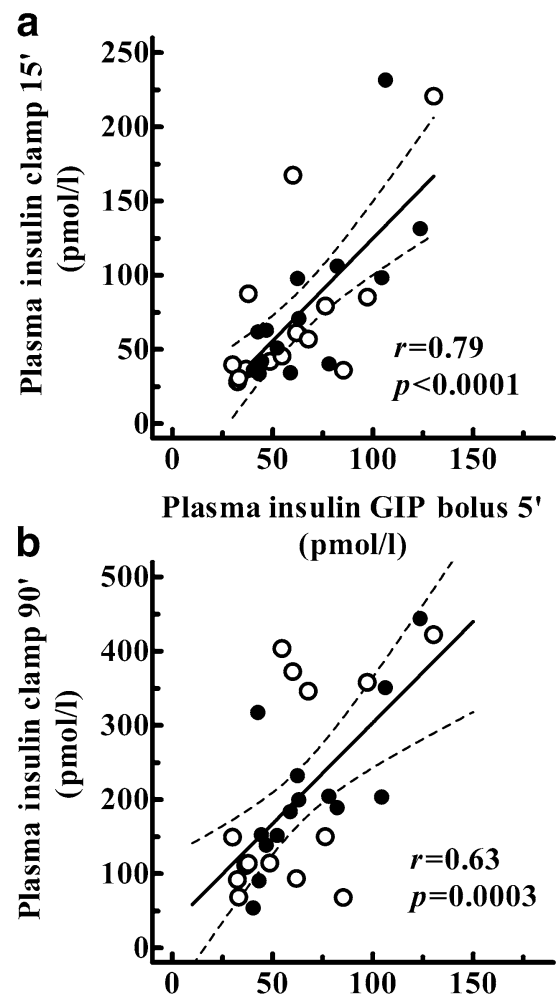

Plasma insulin GIP bolus 5

$(\mathrm{pmol} / \mathrm{l})$

Fig. 6 Correlation analyses between the plasma insulin concentrations measured $5 \mathrm{~min}$ after the bolus administration of GIP (20 $\mathrm{pmol} / \mathrm{kg}$ body weight) and the plasma insulin concentrations measured during the intravenous administration of glucose alone (after 15 min of the clamp experiment; a) and the combined stimulation with intravenous glucose and GIP (after 90 min of the clamp experiment; b) in 14 women with a history of gestational diabetes (filled symbols) and 14 control women (open symbols). $r$, correlation coefficient, calculated by linear regression analysis 


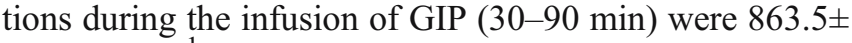
$114.2 \mathrm{mU}^{-1} \mathrm{~min}$ in the women with pGDM and $860.6 \pm$ $168.9 \mathrm{mU} \mathrm{l}^{-1} \mathrm{~min}$ in the control women $(p=0.99)$. For C-peptide, the integrated AUCs during this period were $112.1 \pm 12.0$ and $99.0 \pm 16.9 \mathrm{ng} \mathrm{ml}^{-1} \mathrm{~min}$, respectively $(p=0.53)$.

\section{Regression analyses}

The insulinogenic index calculated 15 min after starting the hyperglycaemic clamp was used to indicate the acute insulin response to glucose stimulation. A hyperbola-like relationship was found between this index and the degree of insulin sensitivity, as derived from the Matsuda index $(r=0.44$; Fig. 5a). This association was not different between the women with pGDM and the control women (Fig. 5a). A similar non-linear inverse relationship was apparent between the insulin concentrations measured 5 min after the GIP bolus administration and the values of the Matsuda index $(r=0.67)$. Again, the slope of the regression line was not different between the groups (Fig. 5b).

The insulin concentrations measured $5 \mathrm{~min}$ after the GIP bolus administration were closely related to those measured during intravenous glucose administration (clamp $\left.15^{\prime} ; r=0.79 ; p<0.0001\right)$ or during the combined administration of glucose and GIP (clamp 90'; $r=0.63 ; p=0.0003$ ). These relations were similar in the two groups (Fig. 6).

There was also a strong linear relationship between the insulin secretory response to GIP bolus administration and the body mass index (details not shown). Interestingly, this association was much stronger in the women with pGDM than in the control women $(r=0.90, p<0.0001$ and $r=0.50$, $p=0.025$, respectively).

\section{Discussion}

Although a reduced insulinotropic effect of GIP in patients with type 2 diabetes has uniformly been reported by different investigators $[7,8,11,24-26]$, the underlying defects are as yet unknown. Indeed, if the action of GIP in type 2 diabetes was impaired as a result of a specific defect, e.g. expression or action of the GIP receptor [27], this may be an interesting target for the development of novel hypoglycaemic agents. Assuming a primary defect in GIP action, we would expect the insulinotropic effect of GIP to be impaired in subjects with a genetic background of type 2 diabetes as well. Consistent with this hypothesis, we recently described a reduced insulinotropic effect of GIP, not only in patients with manifest type 2 diabetes but also in around 50\% of their first-degree relatives, when studied under hyperglycaemic clamp conditions [11]. However, since glucose-stimulated insulin secretion is also impaired in first-degree relatives [15, 28], it was difficult to distinguish between defects in insulin secretion that are specific for the action of GIP and those that are a result of a general impairment in the beta cell function. Therefore, in the present study, a cohort of women with a history of gestational diabetes was first studied under euglycaemic fasting conditions. To compare the responsiveness of insulin secretion to GIP at basal and elevated glucose levels, 14 women in each group were restudied under hyperglycaemic clamp conditions. We report that the insulinotropic effect of GIP is preserved in these women, both under basal euglycaemic conditions and hyperglycaemic conditions, suggesting that a diminished effect of GIP on pancreatic beta cells is unlikely to confer the risk of developing type 2 diabetes in these individuals.

One question arising from the present data is why the insulinotropic effect of GIP was impaired in the first-degree relatives of patients with type 2 diabetes [11] but not in the women with pGDM in the present experiments. The most likely explanation is that diminished GIP-induced insulin secretion in the first-degree relatives was a result of a general impairment in beta cell function. Indeed, these subjects had impaired insulin secretion, not only in response to GIP but also during the intravenous administration of glucose alone [11]. In contrast, all measures of beta cell function in the women with pGDM revealed normal values. Thus, a reduced insulinotropic effect of GIP appears to coincide with a general reduction in beta cell function. This hypothesis is also consistent with our recent observation that the dose-response relationship for the insulinotropic effect of GIP is almost preserved in patients with type 2 diabetes, although this is at generally lower levels of beta cell function [29]. Moreover, the fact that impairments in GIP-induced insulin secretion not only occur in type 2 diabetes but also in other types of diabetes, including MODY 3, latent autoimmune diabetes in adults, early type 1 diabetes and diabetes secondary to chronic pancreatitis, strongly argues against a primary defect in GIP action in the pathogenesis of type 2 diabetes [30].

One intriguing observation from the present study is that insulin secretion in response to GIP increases with higher levels of insulin resistance and obesity. This indicates that defects in insulin action can be compensated for by increased insulin secretion, not only in response to glucose but also in response to GIP and, potentially, other insulin secretagogues. This would also explain the increased insulin responses to GIP administration recently reported in another group of first-degree relatives, who, unlike our previous cohort of subjects, showed a marked impairment in insulin action [31].

A second characteristic defect of the entero-insular axis in patients with type 2 diabetes is a diminished secretion of GLP-1 $[9,10]$. In the present study, based on the same radioimmunoassays as in the previous studies [10], no differences in the secretion of incretin hormones after oral glucose ingestion were found in women with a history of gestational diabetes (Fig. 2). These findings are consistent with previous data from our group and others, demonstrating preserved or even increased (in the case of GIP) secretion of incretin hormones in first-degree relatives of patients with type 2 diabetes $[32,33]$ as well as in subjects with impaired glucose tolerance [34]. However, the present data are at variance with one recent study that reported an impairment of around $20 \%$ in early (0-30 min) GLP-1 
response to oral glucose but no alteration in overall (0-120 min) GLP-1 secretion in a group of women with pGDM [35]. The differences between this and the present study may be a result of the different degrees of metabolic alteration in the pGDM subjects included. Indeed, glycaemic excursions following ingestion of oral glucose in these women were much higher than those in the women included in this study. Therefore, the minor reduction in GLP1 secretion observed in this study may well be secondary to other metabolic disturbances in the pathogenesis of type 2 diabetes.

Some caution should be taken regarding the number of subjects included in this study: while the invasive character and the high experimental effort associated with these studies did not justify testing a larger cohort, it is theoretically possible that minor differences in GIP-induced insulin secretion were overlooked owing to the limited number of participants.

One prominent difference between the women with pGDM and the control subjects in this study was the degree of obesity. This suggests that in this specific group of women with pGDM, obesity was, at least in part, responsible for the observed disturbances in glucose homeostasis. Moreover, the responsiveness of insulin secretion to GIP administration significantly increased with body mass index, particularly in the women with pGDM. Therefore, increased insulin secretion in response to GIP may contribute to the compensation of beta cell function for obesity in women with a history of gestational diabetes.

The modest increase in blood glucose levels $(\sim 2 \mathrm{mg} / \mathrm{dl}$ $[0.1 \mathrm{mmol} / \mathrm{l}])$, despite an increase in insulin secretion observed after GIP bolus administration, is surprising. Probably this was a result of the glucagonotropic effect of GIP, which could influence hepatic glucose release [36]. Alternatively, other glucoregulatory hormones, e.g. cortisole or epinephrine, may also be influenced by GIP administration.

Abnormalities in beta cell function as well as in insulin action have been described in other cohorts of women with a history of gestational diabetes [15, 37-42]. The heterogeneity between these studies is probably a result of the different ethnic and geographic origins of the populations studied. Recently, Buchanan proposed the concept of a failure in beta cell compensation for increasing insulin resistance in a large cohort of women with a Hispanic background [42]. It was suggested that the ambient degree of insulin sensitivity should be taken into account for the accurate assessment of beta cell function [42, 43]. The group of women with pGDM included in the present study was characterised by higher levels of insulin resistance at normal levels of beta cell function. The similar inverse negative relation between glucose-stimulated insulin secretion and the Matsuda index for insulin sensitivity in both groups confirmed the absence of a major defect in beta cell function in the women with pGDM (Fig. 5). Interestingly, a similar hyperbola-like relationship was found between the Matsuda index and the insulin secretory response to the GIP bolus administration. This indicates that insulin secretion in response to GIP and glucose increases to compensate for higher levels of insulin resistance.
In conclusion, the secretion of incretin hormones and the insulinotropic effect of GIP are normal in women with a history of gestational diabetes. Therefore, an early defect in GIP action does not appear to be a risk factor for subsequent development of diabetes in these individuals. An inverse relationship between insulin resistance and the insulin secretory response to glucose or GIP suggests that beta cell secretory function in response to different stimuli compensates for changes in insulin sensitivity.

Acknowledgements The authors are indebted to Professor Peter Butler, Dr Robert Ritzel and Dr Katrin Hücking for critical discussion and helpful suggestions. Professor Gabriele Bonatz and Professor Arne Jensen are gratefully acknowledged for help with subject recruitment. We also acknowledge the excellent technical assistance of Birgit Baller and Lone Bagger. This study was supported by grants from the Deutsche Forschungsgemeinschaft (DFG; Me 2096/2-1, Ga 386/8-1 and Na 203/6-1), FoRUM (F382-03; to J.J. Meier) and the Deutsche Diabetes Gesellschaft (DDG; to J.J. Meier).

\section{References}

1. Unger RH, Eisentraut AM (1969) Entero-insular axis. Arch Int Med 123:261-266

2. Creutzfeldt W (1979) The incretin concept today. Diabetologia $16: 75-85$

3. Creutzfeldt W, Nauck M (1992) Gut hormones and diabetes mellitus. Diabetes Metab Rev 8:149-177

4. Dupré J, Ross SA, Watson D, Brown JC (1973) Stimulation of insulin secretion by gastric inhibitory polypeptide in man. J Clin Endocrinol Metab 37:826-828

5. Kreymann B, Williams G, Ghatei MA, Bloom SR (1987) Glucagon-like peptide-1 [7-36]: a physiological incretin in man. Lancet 2:1300-1304

6. Nauck M, Stöckmann F, Ebert R, Creutzfeldt W (1986) Reduced incretin effect in type 2 (non-insulin-dependent) diabetes. Diabetologia 29:46-54

7. Nauck MA, Heimesaat MM, Ørskov C et al (1993) Preserved incretin activity of glucagon-like peptide 1 [7-36 amide] but not of synthetic human gastric inhibitory polypeptide in patients with type-2 diabetes mellitus. J Clin Invest 91:301-307

8. Vilsbøll T, Krarup T, Madsbad S, Holst JJ (2002) Defective amplification of the late phase insulin response to glucose by GIP in obese type II diabetic patients. Diabetologia 45:11111119

9. Toft-Nielsen MB, Damholt MB, Madsbad S et al (2001) Determinants of impaired secretion of glucagon-like peptide-1 in type 2 diabetic patients. J Clin Endocrinol Metab 86:37173723

10. Vilsbøll T, Krarup T, Deacon CF, Madsbad S, Holst JJ (2001) Reduced postprandial concentrations of intact biologically active glucagon-like peptide 1 in type 2 diabetic patients. Diabetes 50:609-613

11. Meier JJ, Hücking K, Holst JJ et al (2001) Reduced insulinotropic effect of gastric inhibitory polypeptide in first-degree relatives of patients with type 2 diabetes. Diabetes 50: $2497-2504$

12. Meier JJ, Nauck MA, Schmidt WE, Gallwitz B (2002) Gastric Inhibitory Polypeptide (GIP): the neglected incretin revisited. Regul Pept 107:1-13

13. Kjos SL, Buchanan TA (1999) Gestational diabetes. N Engl J Med 341:1749-1756

14. Buchanan TA, Xiang AH, Kjos SL et al (1999) Antepartum predictors of the development of type 2 diabetes in Latino women 11-26 months after pregnancies complicated by gestational diabetes. Diabetes 48:2430-2436 
15. Gerich JE (1998) The genetic basis of type 2 diabetes mellitus: impaired insulin secretion versus impaired insulin sensitivity. Endocr Rev 19:491-503

16. Meier JJ, Nauck MA, Siepmann N et al (2003) Similar insulin secretory response to a GIP bolus injection at euglycemia in first-degree relatives of patients with type 2 diabetes and control subjects. Metabolism 52:1579-1585

17. Deacon CF, Johnsen AH, Holst JJ (1995) Degradation of glucagon-like peptide-1 by human plasma in vitro yields an Nterminally truncated peptide that is a major endogenous metabolite in vivo. J Clin Endocrinol Metab 80:952-957

18. Deacon CF, Nauck MA, Meier JJ, Hücking K, Holst JJ (2000) Degradation of endogenous and exogenous gastric inhibitory polypeptide (GIP) in healthy and in type 2 diabetic subjects as revealed using a new assay for the intact peptide. J Clin Endocrinol Metab 85:3575-3581

19. Matthews DR, Hosker JP, Rudenski AS et al (1985) Homeostasis model assessment: insulin resistance and $\beta$-cell function from fasting plasma glucose and insulin concentrations in man. Diabetologia 28:412-419

20. Matsuda M, DeFronzo RA (1999) Insulin sensitivity indices obtained from oral glucose tolerance testing. Diabetes Care 22: $1462-1470$

21. Kirwan JP, Huston-Presley L, Kalhan SC, Catalano PM (2001) Clinical useful estimates of insulin sensitivity during pregnancy. Diabetes Care 24:1602-1607

22. Seltzer HS, Allen W, Herron AL, Brennan MT (1967) Insulin secretion in response to glycemic stimulus: relation of delayed initial release to carbohydrate intolerance in mild diabetes mellitus. J Clin Invest 46:323-334

23. Expert Committee on the Diagnosis and Classification of Diabetes Mellitus (1997) Report of the Expert Committee on the diagnosis and classification of diabetes mellitus. Diabetes Care 20:1183-1197

24. May JM, Williams RH (1978) The effect of endogenous Gastric Inhibitory Polypeptide on glucose-induced insulin secretion in mild diabetes. Diabetes 27:849-855

25. Krarup T, Saurbrey N, Moody AJ, Kühl C, Madsbad S (1988) Effect of porcine gastric inhibitory polypeptide on $\beta$-cell function in type I and type II diabetes mellitus. Metabolism 36:677682

26. Jones IR, Owens DR, Moody AJ et al (1987) The effects of glucose-dependent insulinotropic polypeptide infused at physiological concentrations in normal subjects and type 2 (noninsulin-dependent) diabetic patients on glucose tolerance and B-cell secretion. Diabetologia 30:707-712

27. Holst JJ, Gromada J, Nauck MA (1997) The pathogenesis of NIDDM involves a defective expression of the GIP receptor. Diabetologia 40:984-986

28. O'Rahilly SP, Nugent Z, Rudenski AS et al (1986) Beta-cell dysfunction, rather than insulin insensitivity, is the primary defect in familial type 2 diabetes. Lancet 16:360-364

29. Meier JJ, Gallwitz B, Kask B et al (2004) Stimulation of insulin secretion by intravenous bolus injection and continuous infusion of gastric inhibitory polypeptide in patients with type 2 diabetes and healthy control subjects. Diabetes 53(Suppl 3): 220-224
30. Vilsbøll T, Knop FK, Krarup T et al (2003) The pathophysiology of diabetes involves a defective amplification of the late-phase insulin response to glucose by glucose-dependent insulinotropic polypeptide-regardless of etiology and phenotype. J Clin Endocrinol Metab 88:4897-4903

31. Rudovich NN, Rochlitz HJ, Pfeiffer AF (2004) Reduced hepatic insulin extraction in response to gastric inhibitory polypeptide compensates for reduced insulin secretion in normalweight and normal glucose tolerant first-degree relatives of type 2 diabetic patients. Diabetes 53:2359-2365

32. Nauck MA, El-Ouaghlidi A, Gabrys B et al (2004) Secretion of incretin hormones (GIP and GLP-1) and incretin effect after oral glucose in first-degree relatives of type 2 diabetic patients. Regul Pept 122:209-217

33. Nyholm B, Walker M, Gravholt CH et al (1999) Twenty-fourhour insulin secretion rates, circulating concentrations of fuel substrates and gut incretin hormones in healthy offspring of type II (non-insulin-dependent) diabetic parents: evidence of several aberrations. Diabetologia 42:1314-1323

34. Rask E, Olsson T, Soderberg S et al (2004) Insulin secretion and incretin hormones after oral glucose in non-obese subjects with impaired glucose tolerance. Metabolism 53:624-631

35. Forbes S, Moonan M, Robinson S et al (2005) Impaired circulating glucagon-like peptide-1 response to oral glucose in women with previous gestational diabetes. Clin Endocrinol (Oxf) 62:51-55

36. Meier JJ, Gallwitz B, Siepmann N et al (2003) Gastric inhibitory polypeptide (GIP) dose-dependently stimulates glucagon secretion in healthy human subjects at euglycaemia. Diabetologia 46:798-801

37. Ward W, Johnston C, Beard J et al (1985) Insulin resistance and impaired insulin secretion in subjects with histories of gestational diabetes. Diabetes 34:861-869

38. Ryan EA, Imes S, Liu D et al (1995) Defects in insulin secretion and action in women with a history of gestational diabetes. Diabetes 44:506-512

39. Efendic S, Hanson V, Persson B, Wajngot A, Luft R (1987) Glucose tolerance, insulin release and insulin sensitivity in normal-weight women with previous gestational diabetes. Diabetes 36:413-419

40. Xiang AH, Peters RK, Trigo E et al (1999) Multiple metabolic defects during late pregnancy in women at high risk for type 2 diabetes. Diabetes 48:848-854

41. Kousta E, Lawrance NJ, Godsland IF et al (2003) Insulin resistance and $\beta$-cell dysfunction in normoglycemic European women with a history of gestational diabetes. Clin Endocrinol 59:289-297

42. Buchanan TA (2001) Pancreatic B-cell defects in gestational diabetes: implications for the pathogenesis and prevention of type 2 diabetes. J Clin Endocrinol Metab 86:989-993

43. Bergman RN (1989) Lilly lecture 1989: towards physiological understanding of glucose tolerance. Minimal-model approach. Diabetes 38:1512-1527 